\title{
Influence of frictional mechanism on chatter vibrations in the cutting process-analytical approach
}

\author{
Andrzej Weremczuk ${ }^{1} \cdot$ Rafal Rusinek $^{1}$ (i)
}

Received: 12 July 2016 / Accepted: 21 September 2016 / Published online: 7 October 2016

(C) The Author(s) 2016. This article is published with open access at Springerlink.com

\begin{abstract}
The paper examines a nonlinear one-degree-offreedom model of the cutting process. The classical regenerative mechanism of chatter is enriched by an additional friction phenomenon which generates frictional chatter. Additionally, the nonlinear cubic stiffness of the tool is taken into account. The aim of the paper is to investigate interactions between regeneration and the frictional effect. The proposed model is solved by the multi-time scale method. The cutting process stability (trivial solution) is determined in order to produce stability lobe diagrams and determine the influence of friction on the process. Finally, the maps of chatter amplitudes are presented and new frictional stability lobe diagrams are proposed to analyse the influence of friction.
\end{abstract}

Keywords Frictional chatter - Regenerative chatter . Cutting process

\section{Introduction}

Nowadays the cutting process is still one of the most popular manufacturing methods. Given increased industrial competition, the manufacturers must reduce costs and improve dimensional accuracy. The efficiency of a machining operation is determined by the metal removal rates, cycle time, machine down time and tool wear. The primary factor

Rafal Rusinek

r.rusinek@pollub.pl

Andrzej Weremczuk

a.weremczuk@pollub.pl

1 Lublin University of Technology, Nadbystrzycka 36, 20-618 Lublin, Poland that limits machining process efficiency is a phenomenon called chatter. Chatter is a dynamic instability that can limit material removal rates, cause poor surface finish and even damage the tool or the workpiece. From a historical point of view, the knowledge of machine tool chatter goes back to almost 100 years ago when Taylor first described this phenomenon [1]. Next, Tlusty et al. [2], Tobias [3] and Kudinov $[4,5]$ gave background of the so-called regenerative chatter. This effect has become the most commonly accepted explanation for machine tool chatter. Later, however, another chatter mechanism produced by friction was developed by Grabec [6]. This mechanism, called frictional chatter, can cause interesting phenomena such as deterministic chaos [6-11]. While the frictional mechanism is based on friction between the tool and the workpiece, the regenerative effect is related to the wavy workpiece surface generated by the previous cutting tooth pass. Wiercigroch et al. define four chatter mechanisms [12, 13]. Besides regenerative and frictional chatter, they also report mode coupling and termomechanical mechanisms. Although trace regeneration and friction are very important practical operations, there are few papers which consider regenerative and frictional mechanisms together, for example [14]. Since friction always exists in a real cutting process, excluding this phenomenon would be a too big simplification.

In the literature, the most often discussed operations are orthogonal cutting operations, e.g. turning and milling. As for turning, the governing equation is relatively simple because the tool has one cutting tooth which still is in contact with the workpiece, so the depth of cut is positive $[12,13,15,16]$. In the case of milling, the direction and value of the cutting force change due to rotation of the multiblade tool, and the cutting is interrupted as each tooth enters and leaves the workpiece. Consequently, the resulting equation of motion is non-smooth $[17,18]$. This causes problems 
during numerical and analytical calculations. An analytical solution of nonlinear problems is not exact but approximate and difficult to obtain. Nonetheless, it is frequently used due to its universality [19]. Sometimes, the impact of ploughing mechanism on chatter stability is presented as well [20].

Recently, scientists pay attention on dynamics of cutting process where multifunctional tools [21] and tools for special operations e.g. thread milling [22] are used. Moreover, the problem of stability lobes in milling process with multiple modes is analysed [23]. In this paper, the useful method of the lowest envelop stability lobes is developed.

In order to get knowledge about the influence of frictional chatter on regenerative chatter and complete field of mathematical approach, the method of time multi-scales is applied here. An explanation of interactions between the frictional and regenerative mechanisms is the main aim of the paper. Therefore, the dynamics of a one-degree-offreedom model of the cutting process is examined. Special attention is devoted to the stability problem of trivial and non-trivial solutions and their dependence on system parameters. Finally, some practical conclusions regarding the cutting process are drawn from the results.

\section{Mathematical model}

For the purpose of analysing the regenerative and frictional mechanisms of chatter, a one-degree-of-freedom model of orthogonal cutting is presented in Fig. 1. In order to explain interactions between the regenerative and frictional mechanisms, only the feed direction $(x)$ is considered here. From our point of view, the feed direction is more important, particularly because the regenerative mechanism depends on tool position in the $x$ (feed) direction and friction between the tool and the chip. The tool is modelled as a lumped mass which is suspended with a nonlinear spring and a linear (viscous) damper. The nonlinear spring is sometimes used in the literature (e.g. $[19,24])$ to model the nonlinear properties of the tool and tool holder, although a linear spring



Fig. 1 Model of orthogonal cutting is more popular. The differential equation of tool motion is presented as

$$
\begin{gathered}
m \ddot{x}_{1}(t)+c \dot{x}_{1}(t)+k_{1} x_{1}^{3}(t)+k x_{1}(t)=K_{r} w . \\
\left(h_{o}-x_{1}(t)+x_{1}(t-\tau)\right)+K_{t}\left(\operatorname{sgn}\left(v_{r}\right)-a_{r} v_{r}+b_{r} v_{r}^{3}\right)
\end{gathered}
$$

where, $m$ is the tool mass, $c$ is damping, $k$ and $k_{1}$ are the linear and nonlinear stiffness coefficients, $w$ is the width of cut, and $h_{o}$ is the initial depth of cut. $K_{r}$ is the regenerative component of the specific cutting force which is related to material shearing (regenerative effect), while $K_{t}$ is the frictional component of the specific cutting force. Dividing Eq. 1 by $m$ and introducing the non-dimensional coordinate $(x)$ and time, after some calculations the non-dimensional spring and damping forces $\left(F_{s}\right.$ and $\left.F_{d}\right)$ are expressed as

$$
\begin{aligned}
& \left.F_{s}=\gamma x^{3}(t)+\omega_{0}^{2} x(t)\right) \\
& F_{d}=\delta \dot{x}(t)
\end{aligned}
$$

The delay differential equation of motion can be presented in a non-dimensional form as

$$
\begin{array}{r}
\ddot{x}(t)+\delta \dot{x}(t)+\gamma x^{3}(t)+\omega_{0}^{2} x(t) \\
=\alpha\left(h_{o}-x(t)+x(t-\tau)\right)+\beta\left(\operatorname{sgn}\left(v_{r}\right)-a_{r} v_{r}+b_{r} v_{r}^{3}\right)
\end{array}
$$

Despite the fact that the regenerative effect is the main cause of chatter, one cannot neglect friction phenomena between the tool and the workpiece as well as between the chip and the tool. Therefore, the present model of the cutting force has two components. A regenerative force, which occurs when the favourable phase develops between the inner and outer modulations, and a friction force between the tool and the workpiece. Then, $\alpha$ denotes the cutting resistance of the regenerative force (regenerative force factor) while $\beta$ is the cutting resistance of the friction force component (friction force factor). In other words, $\alpha$ and $\beta$ tell us how strong the regenerative and the friction components are. The regenerative force depends on the depth of cut $\left(h_{o}\right)$, the present tool position $x(t)$ and the previous position $x(t-\tau)$. In turn, the time delay $x(\tau)$ is connected with the spindle speed $\Omega$ by the equation

$\Omega=\frac{2 \pi}{\tau}$

The friction force depends on the relative velocity $\left(v_{r}\right)$ between the tool and the workpiece (chip) which is expressed as

$v_{r}=v_{c}-\dot{x}(t), \quad v_{c}=d / \tau$

where $v_{c}$ means the cutting speed which also depends on the time delay $\tau$ and a workpiece or a tool diameter $d$. The coefficients $a_{r}$ and $b_{r}$ are responsible for the friction force characteristic presented in Fig. 2. The shape of this characteristic is consistent with the experimental results reported 


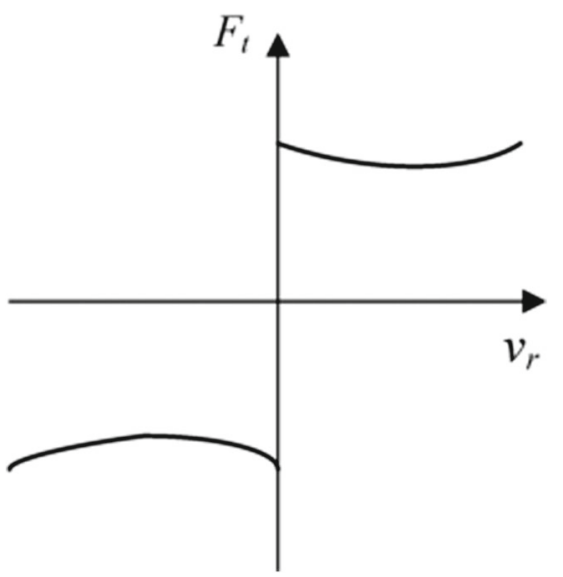

Fig. 2 Friction force characteristic

in [25-29]. The relative velocity $v_{r}$ can be positive and negative. Therefore, the friction force characteristic has two branches.

\section{Analytical solution of chatter vibrations}

The non-dimensional equation of motion of the cutting tool (3) is solved analytically by the multiple scale method [30]. At the beginning, it is assumed that the relative velocity $\left(v_{r}\right)$ is still positive and equals 1 . Next, two scales-the fast $T_{o}$ and the slow $T_{1}$ are introduced and defined as follows:

$T_{0}=t, T_{1}=\varepsilon t$

Then, a solution in the first-order approximation has the form:

$$
\begin{aligned}
x(t) & =x_{0}\left(T_{0}, T_{1}\right)+\varepsilon x_{1}\left(T_{0}, T_{1}\right) \\
x(t-\tau) & =x_{\tau}=x_{0 \tau}\left(T_{0}, T_{1}\right)+\varepsilon x_{1 \tau}\left(T_{0}, T_{1}\right)
\end{aligned}
$$

It is assumed that:

$\omega_{0}^{2}=\omega^{2}+\varepsilon \sigma, \delta=\varepsilon \hat{\delta}, \gamma=\varepsilon \hat{\gamma}, \alpha=\varepsilon \hat{\alpha}, \beta=\varepsilon \hat{\beta}$

where $\varepsilon$ is a formal small parameter. Next, in order to facilitate notation, the tilde is omitted. Using the chain rule, the time derivative is transformed according to the expressions:

$$
\begin{aligned}
\frac{d}{d t} & =\frac{\partial}{\partial T_{0}}+\varepsilon \frac{\partial}{\partial T_{1}} \\
\frac{d^{2}}{d t^{2}} & =\frac{\partial^{2}}{\partial T_{0}^{2}}+\varepsilon \frac{\partial^{2}}{\partial T_{0} \partial T_{1}}+\varepsilon \frac{\partial^{2}}{\partial T_{1} \partial T_{0}}+\ldots \\
& =\frac{\partial^{2}}{\partial T_{0}^{2}}+2 \varepsilon \frac{\partial^{2}}{\partial T_{0} \partial T_{1}}+\ldots
\end{aligned}
$$

Substituting Eqs. 6-9 into Eq. 3 we get:

$$
\begin{aligned}
\frac{\partial^{2} x(t)}{\partial T_{0}^{2}} & +2 \varepsilon \frac{\partial^{2} x(t)}{\partial T_{0} \partial T_{1}}+\varepsilon \beta b_{r}\left(\frac{\partial x(t)}{\partial T_{0}}+\varepsilon \frac{\partial x(t)}{\partial T_{1}}\right)^{3} \\
& -3 \varepsilon \beta b_{r} v_{c}\left(\frac{\partial x(t)}{\partial T_{0}}+\varepsilon \frac{\partial x(t)}{\partial T_{1}}\right)^{2} \\
& +\varepsilon\left(3 \beta b_{r} v_{c}^{2}-\beta a_{r}+\delta\right)\left(\frac{\partial x(t)}{\partial T_{0}}+\varepsilon \frac{\partial x(t)}{\partial T_{1}}\right) \\
& +\varepsilon \alpha\left(\mu x(t)-x_{\tau}(t)-h_{0}\right)+\varepsilon \gamma x(t)^{3} \\
& +\varepsilon \sigma x(t)+\omega^{2} x(t)+\varepsilon \beta\left(a_{r} v_{c}-b_{r} v_{c}^{3}-t_{h}-c\right)=0
\end{aligned}
$$

For clarity, some part of the mathematical derivations is put in the appendix. Finally, we obtain the modulation equations in the form

$$
\begin{aligned}
f_{1}= & a^{\prime}\left(T_{1}\right)=-\frac{1}{2} \delta a\left(T_{1}\right)-\frac{1}{2} \alpha a\left(T_{1}\right) \sin \tau \\
& +\frac{1}{2} \beta a_{r} a\left(T_{1}\right)-\frac{3}{8} \beta b_{r} a\left(T_{1}\right)^{3}-\frac{3}{2} \beta b_{r} v_{c}^{2} a\left(T_{1}\right) \\
f_{2}= & \beta^{\prime}\left(T_{1}\right)=\frac{1}{2} \mu \alpha+\frac{1}{2} \sigma+\frac{3}{8} \gamma a\left(T_{1}\right)^{2}-\frac{1}{2} \alpha \cos \tau
\end{aligned}
$$

Then, for the steady-state solution, Eq. 11 take the form:

$$
\begin{aligned}
-\frac{1}{2} \delta a-\frac{1}{2} \alpha a \sin \tau+\frac{1}{2} \beta a_{r} a-\frac{3}{8} \beta b_{r} a^{3}-\frac{3}{2} \beta b_{r} v_{c}^{2} a & =0 \\
\frac{1}{2} \mu \alpha+\frac{1}{2}\left(\omega_{o}^{2}-\omega^{2}\right)+\frac{3}{8} \gamma a^{2}-\frac{1}{2} \alpha \cos \tau & =0
\end{aligned}
$$

Solving Eq. 12 ,one trivial $\left(a_{1}\right)$ and two non-trivial (periodic) solutions $\left(a_{2}\right)$ are found.

$$
\begin{aligned}
a_{1} & =0 \\
a_{2,3} & =2 \sqrt{\frac{a_{r}-\frac{\delta}{\beta}}{3 b_{r}} \mp \frac{\alpha \sin (\tau)}{3 \beta b_{r}}-\frac{d^{2}}{\tau^{2}}}
\end{aligned}
$$

The trivial solution $\left(a_{1}\right)$ is important from a practical point of view because here the cutting process is stable without chatter vibrations. When the trivial solution is unstable, chatter appears. Therefore, the problem of solution stability is of great importance.

To analyse the stability of steady-state solutions, Eq. 11 are linearised with respect to $a\left(T_{1}\right)$ and $\beta\left(T_{1}\right)$. Next, the Jacobian matrix is defined as

$J=\left(\begin{array}{ll}\frac{d f_{1}}{d a} & \frac{d f_{1}}{d \beta\left(T_{1}\right)} \\ \frac{d f_{2}}{d a} & \frac{d f_{2}}{d \beta\left(T_{1}\right)}\end{array}\right)$

The eigenvalue of the Jacobian (Eq. 14) should have a negative real part in order to produce a stable solution. The 
eigenvalue, which defines stability of trivial and non-trivial solution is expressed as

$\frac{1}{8}\left(4 a_{r} \beta-9 a^{2} b_{r} \beta-12 b_{r} \beta(d / \tau)^{2}-4 \delta-4 \alpha \sin \tau\right)$

Trivial solution stability For the trivial solution $\left(a_{1}=0\right)$, the eigenvalue (Eq. 15) takes the form

$\beta\left(a_{r}-3 b_{r}(d / \tau)^{2}\right)-\delta-\alpha \sin \tau<0$

The stability borders of the trivial solution determine the so-called stability lobe diagram (SLD) which is shown graphically in Fig. 3 assuming the following parameters: $\delta=0.1, \beta=0.8, a_{r}=0.5, b_{r}=0.1$ and $d=1$. The SLD shows the plane of the parameters $\Omega-\alpha$ where cutting process is stable (the trivial solution is stable). This area is white in the SLD while the colour lobes point to the chatter vibration amplitude.

Inside the lobes, the non-trivial (periodic) solution exists. Its amplitude and the lobe width depend on the friction force factor $(\beta)$. At $\beta=0.01$, the chatter region is smaller, but the amplitude is higher approaching even to 30 (Fig. 3a). At stronger friction $(\beta=0.1$ and especially $\beta=0.8)$, the chatter region is wider and the amplitudes of chatter are significantly smaller. Thus, friction broadens the chatter region but limits the vibration amplitude.

Stability of non-trivial solutions The non-trivial (periodic) solutions $\left(a_{2,3}\right)$ are stable when the following equation is satisfied

$\beta\left(a_{r}-3 b_{r}(d / \tau)^{2}\right)-\delta+\alpha\left(\frac{1}{2} \mp \frac{3}{2}\right) \sin \tau>0$

The first periodic solution $a_{2}$ is stable exactly when the trivial solutions is unstable, but the second non-trivial solution $a_{3}$ is stable in the regions where the trivial solutions is stable. Thus, two solutions: trivial $a_{1}$ and periodic $a_{3}$, can exist in the same region of the SLD depending on the initial conditions. The same behaviour observed for the nonlinear regenerative model is reported in [31]. Both periodic solutions $\left(a_{2}\right.$ and $\left.a_{3}\right)$ are presented in Fig. 4. Interestingly, that in the first-order approximation chatter vibrations do not depend on cubic nonlinearity determined by the $\gamma$ coefficient. Probably the solution of the second order approximation reveals the influence of $\gamma$ on the system's dynamics. Similar diagrams with unstable lobes are obtained on the plane $\Omega-\beta$ (Fig. 5). In this case, three variants of the coefficient of regenerative effect $(\alpha)$ are analysed $\alpha=0.01, \alpha=0.1$ and $\alpha=0.4$. For $\alpha=0.01$ (Fig. 5a) there is a critical value of $\beta=0.2$. This critical $\beta$ means that, below this value, the cutting process is free of chatter regardless of $\omega$. Unstable lobes are hardly visible because the whole region $\beta>0.2$ is unstable. In other words, the a

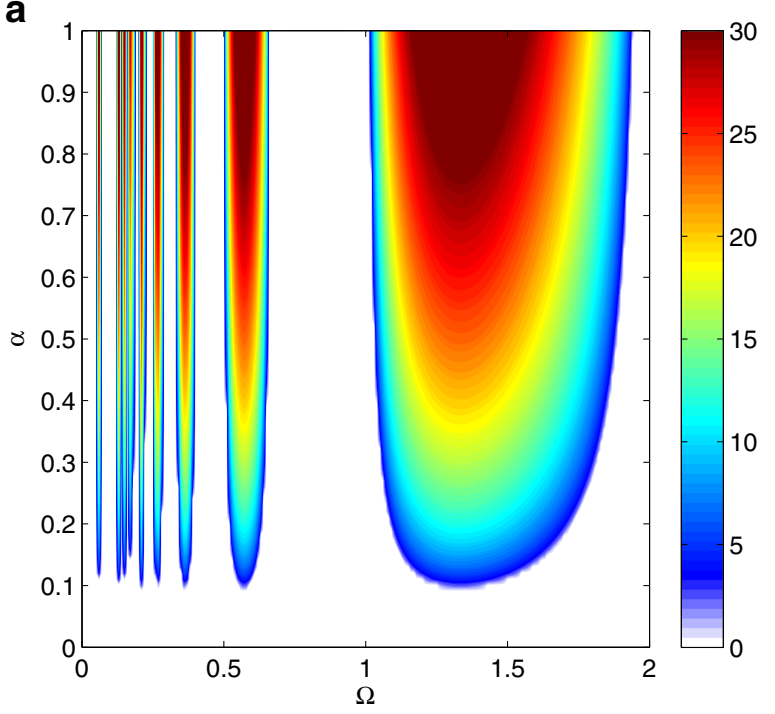

b

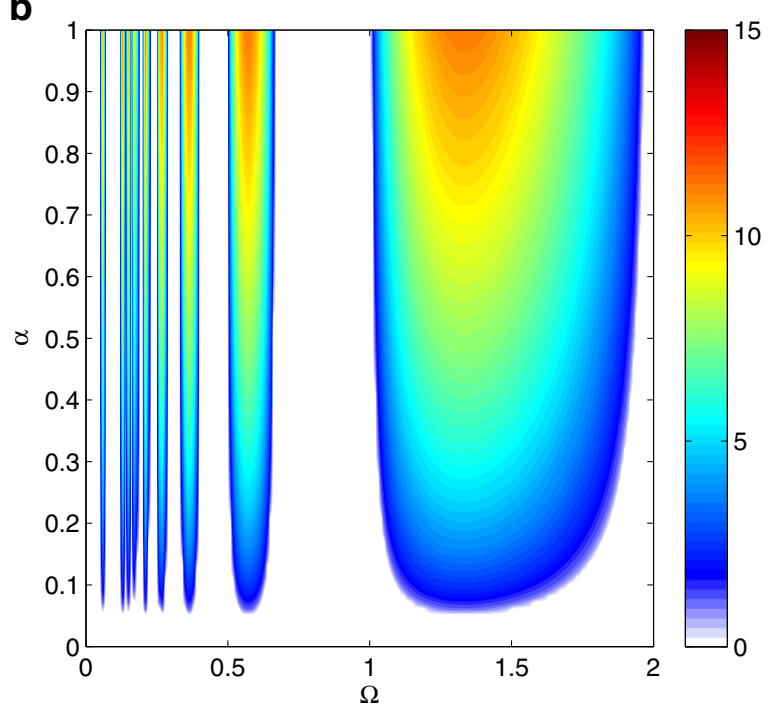

C

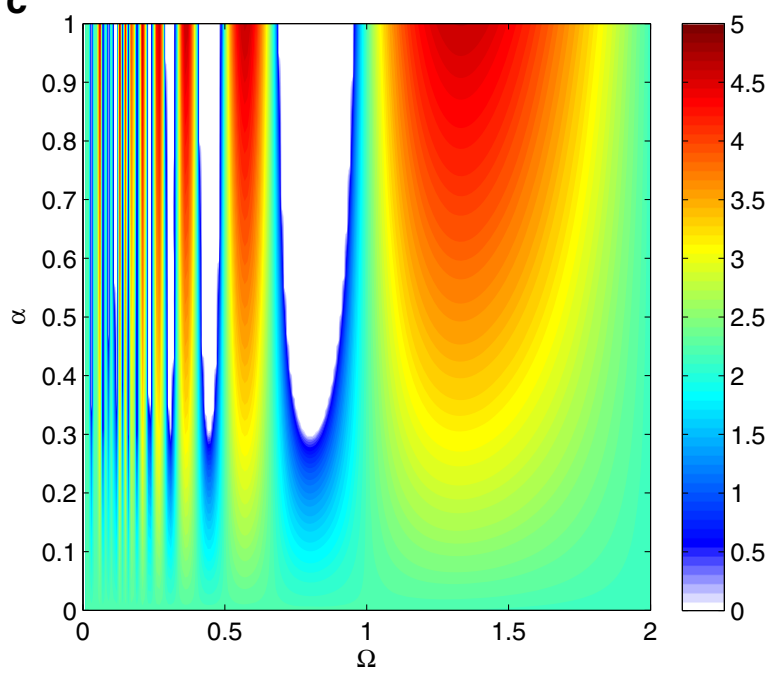

Fig. 3 Stability lobe diagrams. Influence of regeneration mechanism $(\beta)$ on stability of trivial solution for $\beta=0.01(\mathbf{a}), \beta=0.1(\mathbf{b})$ and $\beta=0.8(\mathbf{c})$ 

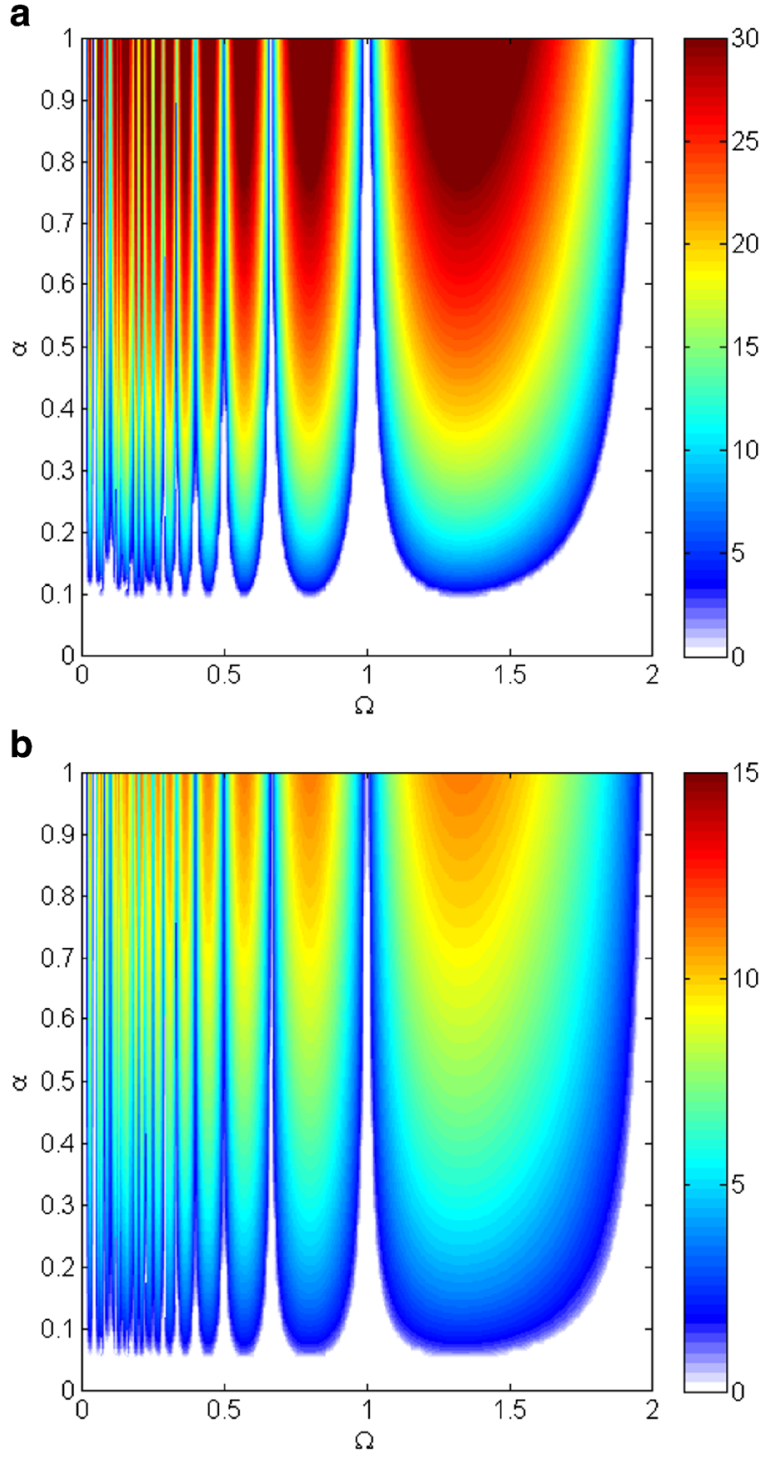

C



Fig. 4 Stability lobe diagrams. Influence of regeneration mechanism $(\beta)$ on stability of non-trivial solution for $\beta=0.01(\mathbf{a}), \beta=0.1$ (b) and $\beta=0.8$ (c)

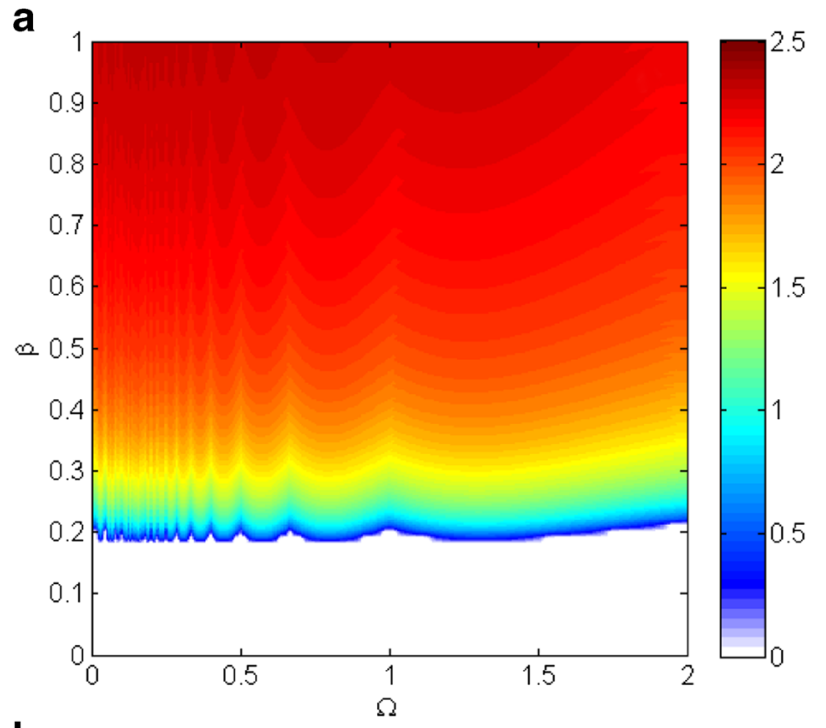

b

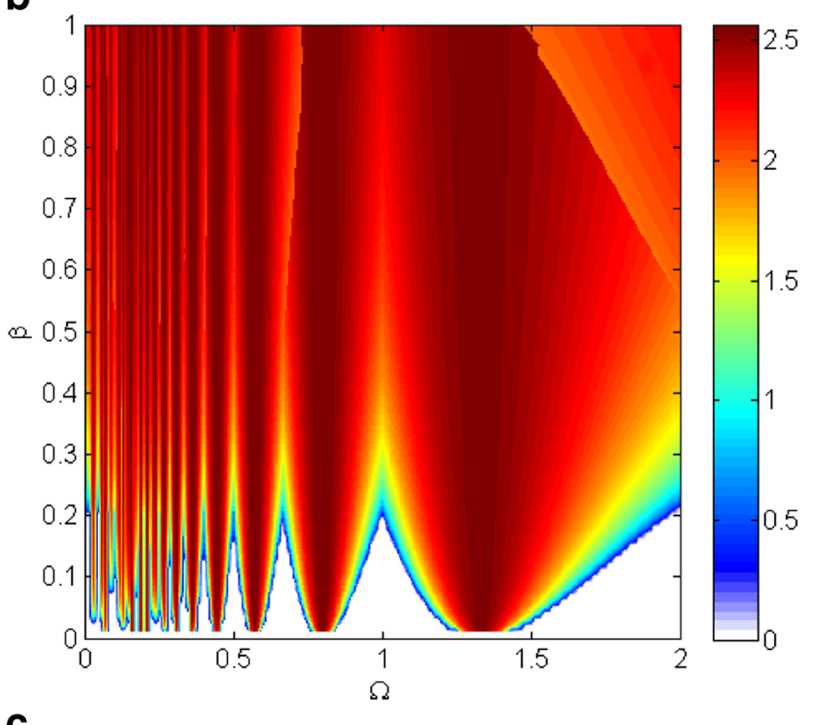

C

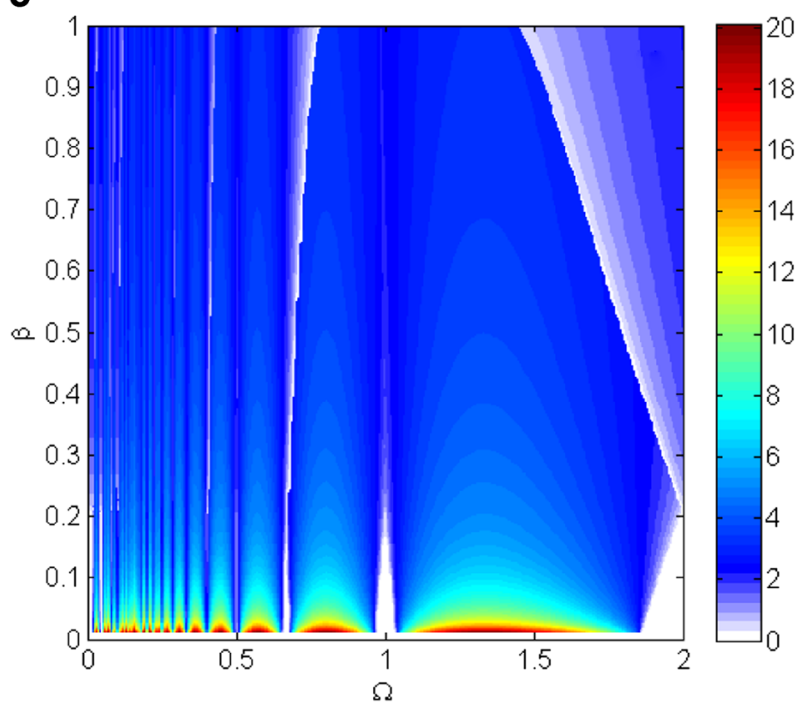

Fig. 5 Influence of friction $(\beta)$ on stability of non-trivial solution for $\alpha=0.01(\mathbf{a}), \alpha=0.1(\mathbf{b})$ and $\alpha=0.4(\mathbf{c})$ 
periodic solutions are stable. Unstable lobes are more visible when $\alpha=0.1$ (Fig. 5b). In the analysed system, the most interesting behaviour can be observed for $\alpha=0.4$ (Fig. 5c). The highest amplitudes occur for the small $\beta$ and unstable lobes seem to be inverted. Here, the regenerative mechanism dominates over the frictional one.

\section{Discussion and conclusions}

Chatter vibrations as a result of classical regenerative and extra frictional mechanisms are investigated here with respect to interactions between them. The analytical method of multiple time scales is used successfully to solve the nonlinear problem of the cutting process. Although the nonlinear properties of the tool stiffness are assumed, their influence on cutting dynamics is not allowed for in the firstorder approximation. Probably, the second order approximation would be better to this aim; however, the influence of the frictional mechanism on regenerative chatter is visible. Classical unstable lobes generated by the regenerative effect are modified by the action of friction. The friction phenomenon widens unstable cutting regions, but on the other hand, it reduces the chatter vibration amplitude. The regenerative model of cutting with friction has trivial and two periodic (non-trivial) solutions. The periodic and trivial solutions can exists simultaneously at specific cutting speeds because both solutions can be stable. From practical point of view it means that any disturbance causing a change of initial conditions can lead to chatter even in the region where the classical regenerative cutting process should be stable, this is, for a small $\alpha$. Such a change of initial conditions can be caused for example by chip break. The interesting phenomenon of reverse unstable lobes is shown on the plane of rotational speed-friction force coefficient $(\Omega-\beta)$. We can observed an untypical behaviour where the small $\beta$ generates a higher vibration amplitude than large one. The stability diagram on the plane of rotational speed $(\omega)$-friction force component $(\beta)$ is an equivalent of the classical stability lobe diagram (SLD) and can be called a frictional stability lobe diagram-FSLD.

Investigation of friction and regenerative chatter will be continued using the numerical method in order to find aperiodic and irregular vibrations in the nonlinear model of the cutting process. Moreover, experimental tests are planned to be performed in order to verify the theoretical results, and most of all, to obtain the real coefficient of frictional and regenerative force components.

Acknowledgments The work of the first author is financially supported by the National Science Centre under the project no. DEC2013/09/N/ST8/01202. The contribution of the second author is financially supported by the National Science Centre under the project no. DEC-2011/01/B/ST8/07504.
Open Access This article is distributed under the terms of the Creative Commons Attribution 4.0 International License (http:// creativecommons.org/licenses/by/4.0/), which permits unrestricted use, distribution, and reproduction in any medium, provided you give appropriate credit to the original author(s) and the source, provide a link to the Creative Commons license, and indicate if changes were made.

\section{Appendix}

Expanding derivatives of the Eq. 10, we obtain:

$$
\begin{aligned}
& \frac{\partial x(t)}{\partial T_{0}}=\frac{\partial x_{0}}{\partial T_{0}}+\varepsilon \frac{\partial x_{1}}{\partial T_{0}} \\
& \frac{\partial^{2} x(t)}{\partial T_{0}^{2}}=\frac{\partial^{2} x_{0}}{\partial T_{0}^{2}}+\varepsilon \frac{\partial^{2} x_{1}}{\partial T_{0}^{2}} \frac{\partial^{2} x(t)}{\partial T_{0} \partial T_{1}} \\
&=\frac{\partial^{2} x_{0}}{\partial T_{0} \partial T_{1}}+\varepsilon \frac{\partial^{2} x_{1}}{\partial T_{0} \partial T_{1}} \\
& \varepsilon \frac{\partial^{2} x_{1}}{\partial T_{0}^{2}}+\frac{\partial^{2} x_{0}}{\partial T_{0}^{2}}+2 \varepsilon \frac{\partial^{2} x_{0}}{\partial T_{0} \partial T_{1}}+\varepsilon \beta b_{r}\left(\frac{\partial x_{0}}{\partial T_{0}}\right)^{3} \\
&-3 \varepsilon \beta b_{r} v_{c}\left(\frac{\partial x_{0}}{\partial T_{0}}\right)^{2}+\varepsilon\left(3 \beta b_{r} v_{c}^{2}-\beta a_{r}+\delta\right)\left(\frac{\partial x_{0}}{\partial T_{0}}\right)^{2}+\varepsilon \alpha\left(\mu x_{0}-x_{0 \tau}-h_{0}\right)+\varepsilon \gamma x_{0}^{3} \\
&+\varepsilon \beta\left(a_{r} v_{c}-b_{r} v_{c}^{3}-t_{h}-c\right)=0
\end{aligned}
$$

Equating coefficients of powers of $\varepsilon^{0}$ and $\varepsilon^{1}$, we obtain:

$$
\begin{aligned}
\varepsilon^{0} \Rightarrow & \frac{\partial^{2} x_{0}}{\partial T_{0}^{2}}+\omega^{2} x_{0}=0 \\
\varepsilon^{1} \Rightarrow & \frac{\partial^{2} x_{1}}{\partial T_{0}^{2}}+2 \frac{\partial^{2} x_{0}}{\partial T_{0} \partial T_{1}}+\beta b_{r}\left(\frac{\partial x_{0}}{\partial T_{0}}\right)^{3} \\
& -3 \beta b_{r} v_{c}\left(\frac{\partial x_{0}}{\partial T_{0}}\right)^{2}+\left(3 \beta b_{r} v_{c}^{2}-\beta a_{r}+\delta\right)\left(\frac{\partial x_{0}}{\partial T_{0}}\right) \\
& +\alpha\left(\mu x_{0}-x_{0 \tau}-h_{0}\right)+\gamma x_{0}^{3}+\omega^{2} x_{1} \\
& +\sigma x_{0}+\beta\left(a_{r} v_{c}-b_{r} v_{c}^{3}-t_{h}-c\right)=0
\end{aligned}
$$

It is convenient to express the solution of first Eq. 20 in the complex form:

$$
\begin{aligned}
x_{0}\left(T_{0}, T_{1}\right) & =A\left(T_{1}\right) e^{i T_{0}}+\bar{A}\left(T_{1}\right) e^{-i T_{0}} \\
x_{0 \tau}\left(T_{0}, T_{1}\right) & =A\left(T_{1}\right) e^{i\left(T_{0}-\tau\right)}+\bar{A}\left(T_{1}\right) e^{-i\left(T_{0}-\tau\right)}
\end{aligned}
$$

where $\bar{A}$ is the complex conjugate of $A$, which is an arbitrary complex function of $T_{1}$. Substituting Eq. 21 into second Eq. 20 and expanding the derivatives, we get:

$$
\begin{aligned}
\frac{\partial x_{0}}{\partial T_{0}} & =A\left(T_{1}\right) i e^{i T_{0}}-\bar{A}\left(T_{1}\right) i e^{-i T_{0}} \\
\frac{\partial^{2} x_{0}}{\partial T_{0} \partial T_{1}} & =A^{\prime}\left(T_{1}\right) i e^{i T_{0}}-\bar{A}^{\prime}\left(T_{1}\right) i e^{-i T_{0}}
\end{aligned}
$$


and then the following equation is obtained:

$$
\begin{array}{r}
\frac{\partial^{2} x_{1}}{\partial T_{0}^{2}}+\omega^{2} x_{1}+2\left(A^{\prime}\left(T_{1}\right) i e^{i T_{0}}-\bar{A}^{\prime}\left(T_{1}\right) i e^{-i T_{0}}\right) \\
+\beta b_{r}\left(A\left(T_{1}\right) i e^{i T_{0}}-\bar{A}\left(T_{1}\right) i e^{-i T_{0}}\right)^{3} \\
-3 \beta b_{r} v_{c}\left(A\left(T_{1}\right) i e^{i T_{0}}-\bar{A}\left(T_{1}\right) i e^{-i T_{0}}\right)^{2} \\
+\left(3 \beta b_{r} v_{c}^{2}-\beta a_{r}+\delta\right)\left(A\left(T_{1}\right) i e^{i T_{0}}-\bar{A}\left(T_{1}\right) i e^{-i T_{0}}\right) \\
+\alpha \mu\left[A\left(T_{1}\right) e^{i T_{0}}+\bar{A}\left(T_{1}\right) e^{-i T_{0}}\right] \\
-\alpha\left[A\left(T_{1}\right) e^{i\left(T_{0}-\tau\right)}+\bar{A}\left(T_{1}\right) e^{-i\left(T_{0}-\tau\right)}\right]-\alpha h_{0} \\
+\gamma\left(A\left(T_{1}\right) e^{i T_{0}}+\bar{A}\left(T_{1}\right) e^{-i T_{0}}\right)^{3} \\
+\sigma\left(A\left(T_{1}\right) e^{i T_{0}}+\bar{A}\left(T_{1}\right) e^{-i T_{0}}\right) \\
+\beta\left(a_{r} v_{c}-b_{r} v_{c}^{3}-t_{h}-c\right)=0
\end{array}
$$

Ordering Eq. 23, we get its final form

$$
\begin{array}{r}
\frac{\partial^{2} x_{1}}{\partial T_{0}^{2}}+\omega^{2} x_{1}+\left(i \delta A\left(T_{1}\right)+\alpha \mu A\left(T_{1}\right)\right. \\
+\sigma A\left(T_{1}\right)-i \beta a_{r} A\left(T_{1}\right)+3 i \beta b_{r} v_{c}^{2} A\left(T_{1}\right) \\
+3 \gamma A\left(T_{1}\right)^{2} \bar{A}\left(T_{1}\right) \\
\left.+3 i \beta b_{r} A\left(T_{1}\right)^{2} \bar{A}\left(T_{1}\right)+2 i A^{\prime}\left(T_{1}\right)-\alpha A\left(T_{1}\right) e^{-i \tau}\right) e^{i T_{0}} \\
+\left(-i \delta \bar{A}\left(T_{1}\right)+\alpha \mu \bar{A}\left(T_{1}\right)\right. \\
+\sigma \bar{A}\left(T_{1}\right)+i \beta a_{r} \bar{A}\left(T_{1}\right)-3 i \beta b_{r} v_{c}^{2} \bar{A}\left(T_{1}\right) \\
+3 \gamma \bar{A}\left(T_{1}\right)^{2} A\left(T_{1}\right)-3 i \beta b_{r} \bar{A}\left(T_{1}\right)^{2} A\left(T_{1}\right) \\
\left.-2 i \bar{A}^{\prime}\left(T_{1}\right)-\alpha \bar{A}\left(T_{1}\right) e^{-i \tau}\right) e^{-i T_{0}} \\
+\left(\gamma-i \beta b_{r}\right) A\left(T_{1}\right)^{3} e^{3 i T_{0}}+\left(\gamma+i \beta b_{r}\right) \bar{A}\left(T_{1}\right)^{3} e^{-3 i T_{0}} \\
-6 \beta b_{r} v_{c} A\left(T_{1}\right) \bar{A}\left(T_{1}\right) \\
+\beta\left(a_{r} v_{c}-b_{r} v_{c}^{3}-t_{h}-c\right)-\alpha h_{0}=0
\end{array}
$$

The secular term of Eq. 24 vanishes if and only if:

$S T_{1} e^{i T_{0}}=0, S T_{2} e^{-i T_{0}}=0$

where $S T_{1}$ and $S T_{2}$ are the secular generating terms. This leads to the equations:

$$
\begin{array}{r}
i \delta A\left(T_{1}\right)+\alpha \mu A\left(T_{1}\right)+\sigma A\left(T_{1}\right)-i \beta a_{r} A\left(T_{1}\right) \\
+3 i \beta b_{r} v_{c}^{2} A\left(T_{1}\right)+3 \gamma A\left(T_{1}\right)^{2} \bar{A}\left(T_{1}\right) \\
+3 i \beta b_{r} A\left(T_{1}\right)^{2} \bar{A}\left(T_{1}\right)+2 i A^{\prime}\left(T_{1}\right)-\alpha A\left(T_{1}\right) e^{-i \tau}=0 \\
-i \delta \bar{A}\left(T_{1}\right)+\alpha \mu \bar{A}\left(T_{1}\right)+\sigma \bar{A}\left(T_{1}\right)+i \beta a_{r} \bar{A}\left(T_{1}\right) \\
-3 i \beta b_{r} v_{c}^{2} \bar{A}\left(T_{1}\right)+3 \gamma \bar{A}\left(T_{1}\right)^{2} A\left(T_{1}\right) \\
-3 i \beta b_{r} \bar{A}\left(T_{1}\right)^{2} A\left(T_{1}\right)-2 i \bar{A}^{\prime}\left(T_{1}\right)-\alpha \bar{A}\left(T_{1}\right) e^{-i \tau}=0
\end{array}
$$

Substituting into Eq. 26, the polar form of the complex amplitude:

$$
\begin{aligned}
A\left(T_{1}\right) & =\frac{1}{2} a\left(T_{1}\right) e^{i \beta\left(T_{1}\right)} \\
A^{\prime}\left(T_{1}\right) & =\frac{1}{2} a^{\prime}\left(T_{1}\right) e^{i \beta\left(T_{1}\right)}+\frac{1}{2} i a\left(T_{1}\right) \beta^{\prime}\left(T_{1}\right) e^{i \beta\left(T_{1}\right)} \\
\bar{A}\left(T_{1}\right) & =\frac{1}{2} a\left(T_{1}\right) e^{-i \beta\left(T_{1}\right)} \\
\bar{A}^{\prime}\left(T_{1}\right) & =\frac{1}{2} a^{\prime}\left(T_{1}\right) e^{-i \beta\left(T_{1}\right)}-\frac{1}{2} i a\left(T_{1}\right) \beta^{\prime}\left(T_{1}\right) e^{-i \beta\left(T_{1}\right)}
\end{aligned}
$$

results in:

$$
\begin{array}{r}
-\frac{1}{2} \alpha a\left(T_{1}\right) e^{-i \tau+i \beta\left(T_{1}\right)}+\frac{1}{2} i \delta a\left(T_{1}\right) e^{i \beta\left(T_{1}\right)} \\
+\frac{1}{2} \mu \alpha a\left(T_{1}\right) e^{i \beta\left(T_{1}\right)}+\frac{1}{2} \sigma a\left(T_{1}\right) e^{i \beta\left(T_{1}\right)} \\
+\frac{3}{8} \gamma a\left(T_{1}\right)^{3} e^{i \beta\left(T_{1}\right)}-\frac{1}{2} i \beta a_{r} a\left(T_{1}\right) e^{i \beta\left(T_{1}\right)} \\
+\frac{3}{8} i \beta b_{r} a\left(T_{1}\right)^{3} e^{i \beta\left(T_{1}\right)}+\frac{3}{2} i \beta b_{r} v_{c}^{2} a\left(T_{1}\right) e^{i \beta\left(T_{1}\right)} \\
+2 i\left[\frac{1}{2} a^{\prime}\left(T_{1}\right) e^{i \beta\left(T_{1}\right)}+\frac{1}{2} i a\left(T_{1}\right) \beta^{\prime}\left(T_{1}\right) e^{i \beta\left(T_{1}\right)}\right]=0 \\
-\frac{1}{2} \alpha a\left(T_{1}\right) e^{i \tau-i \beta\left(T_{1}\right)}-\frac{1}{2} i \delta a\left(T_{1}\right) e^{-i \beta\left(T_{1}\right)} \\
+\frac{1}{2} \mu \alpha a\left(T_{1}\right) e^{-i \beta\left(T_{1}\right)}+\frac{1}{2} \sigma a\left(T_{1}\right) e^{-i \beta\left(T_{1}\right)} \\
+\frac{3}{8} \gamma a\left(T_{1}\right)^{3} e^{-i \beta\left(T_{1}\right)} \\
+\frac{1}{2} i \beta a_{r} a\left(T_{1}\right) e^{-i \beta\left(T_{1}\right)} \\
-\frac{3}{8} i \beta b_{r} a\left(T_{1}\right)^{3} e^{-i \beta\left(T_{1}\right)} \\
-\frac{3}{2} i \beta b_{r} v_{c}^{2} a\left(T_{1}\right) e^{-i \beta\left(T_{1}\right)} \\
-2 i\left[\frac{1}{2} a^{\prime}\left(T_{1}\right) e^{-i \beta\left(T_{1}\right)}-\frac{1}{2} i a\left(T_{1}\right) \beta^{\prime}\left(T_{1}\right) e^{-i \beta\left(T_{1}\right)}\right]=0
\end{array}
$$

After the transformations of the first Eq. 28, we obtain:

$$
\begin{array}{r}
-\frac{1}{2} \alpha a\left(T_{1}\right) e^{-i \tau}+\frac{1}{2} i \delta a\left(T_{1}\right)+\frac{1}{2} \mu \alpha a\left(T_{1}\right) \\
+\frac{1}{2} \sigma a\left(T_{1}\right)+\frac{3}{8} \gamma a\left(T_{1}\right)^{3}-\frac{1}{2} i \beta a_{r} a\left(T_{1}\right) \\
+\frac{3}{8} i \beta b_{r} a\left(T_{1}\right)^{3}+\frac{3}{2} i \beta b_{r} v_{c}^{2} a\left(T_{1}\right)+i a^{\prime}\left(T_{1}\right) \\
-a\left(T_{1}\right) \beta^{\prime}\left(T_{1}\right)=0
\end{array}
$$

Then recalling

$e^{-i \tau}=\cos \tau-i \sin \tau$ 
The normal form is obtained:

$$
\begin{array}{r}
\frac{1}{2} i \delta a\left(T_{1}\right)+\frac{1}{2} \mu \alpha a\left(T_{1}\right)+\frac{1}{2} \sigma a\left(T_{1}\right)+\frac{3}{8} \gamma a\left(T_{1}\right)^{3} \\
-\frac{1}{2} \alpha a\left(T_{1}\right) \cos \tau+\frac{1}{2} i \alpha a\left(T_{1}\right) \sin \tau \\
-\frac{1}{2} i \beta a_{r} a\left(T_{1}\right)+\frac{3}{8} i \beta b_{r} a\left(T_{1}\right)^{3}+\frac{3}{2} i \beta b_{r} v_{c}^{2} a\left(T_{1}\right) \\
+i a^{\prime}\left(T_{1}\right)-a\left(T_{1}\right) \beta^{\prime}\left(T_{1}\right)=0
\end{array}
$$

Separating the real and imaginary parts, the two, so-called, modulation equations are found:

$$
\begin{array}{r}
\frac{1}{2} \delta a\left(T_{1}\right)+\frac{1}{2} \alpha a\left(T_{1}\right) \sin \tau-\frac{1}{2} \beta a_{r} a\left(T_{1}\right) \\
+\frac{3}{8} \beta b_{r} a\left(T_{1}\right)^{3}+\frac{3}{2} \beta b_{r} v_{c}^{2} a\left(T_{1}\right)+a^{\prime}\left(T_{1}\right)=0 \\
\frac{1}{2} \mu \alpha a\left(T_{1}\right)+\frac{1}{2} \sigma a\left(T_{1}\right)+\frac{3}{8} \gamma a\left(T_{1}\right)^{3} \\
-\frac{1}{2} \alpha a\left(T_{1}\right) \cos \tau-a\left(T_{1}\right) \beta^{\prime}\left(T_{1}\right)=0
\end{array}
$$

\section{References}

1. Taylor F (1907) On the art of cutting metal. Trans ASME 28:31248

2. Tlusty J, Polacek M (eds) (1963) Stability of machine tools against self-excited vibration in machining, Proc ASME Prod Eng Res Conf

3. Tobias SA (1965) Machine tool vibration. John Wiley, New York and USA

4. Kudinov VA (1955) Theory of vibration generated from metal cutting. New Technology of Mechanical Engineering:1-7

5. Kudinov VA (1963) Dynamics characteristics of the metal cutting process. Stanki i Instrument 10:1-7

6. Grabec I (1986) Chaos generated by the cutting process. Phys Lett A A117:384-386

7. Grabec I (1998) Chaotic dynamics of the cutting process. Int J Mach Tools Manuf 28(1):19-32

8. Lipski J, Litak G, Rusinek R, Szabelski K, Teter A, Warminski J, Zaleski K (2002) Surface quality of a work material's influence on the vibrations of the cutting process. J Sound Vib 252(4):739_ 737

9. Rusinek R, Wiercigroch M, Wahi P (2014) Influence of tool flank forces on complex dynamics of cutting process. Int J Bifurcation Chaos 24(09):1450115

10. Rusinek R, Wiercigroch M, Wahi P (2014) Modelling of frictional chatter in metal cutting. Int J Mech Sci 89:167-176

11. Wiercigroch M (1997) Chaotic vibration of a simple model of the machine tool-cutting process system. J Vib Acoust 119:468475
12. Wiercigroch M, Budak E (2001) Sources of nonlinearities, chatter generation and suppression in metal cutting. Philos Trans R Soc A Math Phys Eng Sci 359(1781):663-693

13. Wiercigroch M, Krivtsov AM (2001) Frictional chatter in orthogonal metal cutting. Phil Trans The Royal Society of London A Mathematical Physical And Engineering Science 359:713-738

14. Rusinek R, Kecik K, Warminski J, Weremczuk A (2011) Dynamic model of cutting process with modulated spindle speed. AIP Conf Proc: $805-809$

15. Gradisek J, Grabec I, Siegert S, Friedrich R (2002) Stochastic dynamics of metal cutting: bifurcation phenomena in turning. Mech Syst Signal Process 16(5):831-840

16. Kalmar-Nagy T, Wahi P (2008) Towards practical stability limits in Turning. In: Proceedings of the 6th EUROMECH Nonlinear Oscillations Conference St. Petersburg, Russia

17. Stepan G (2001) Modelling nonlinear regenerative effect in metal cutting. Philos Trans R Soc A Math Phys Eng Sci 359:739-757

18. Stepan G, Szalai R, Insperger T (2004) Nonlinear dynamics of high-speed milling subjected to regenerative effect. In: Radons G, Neugebauer R (eds) Nonlinear Dynamics of Production Systems 111-127. Wiley-VCH, Weinheim

19. Nayfeh AH, Chin CM, Pratt J (1997) Perturbation methods in nonlinear dynamics-applications to machining dynamics. J Manuf Sci Eng 119:485-493

20. Wan M, Ma YC, Feng J, Zhang WH (2016) Study of static and dynamic ploughing mechanisms by establishing generalized model with static milling forces. Int J Mech Sci 114:120-131

21. Wan M, Murat Kilic Z, Altintas Y (2015) Mechanics and dynamics of multifunctional tools. J Manuf Sci Eng 137(1):011019

22. Wan M, Altintas Y (2014) Mechanics and dynamics of thread milling process. Int J Mach Tools Manuf 87:16-26

23. Wan M, Ma YC, Zhang WH, Yang Y (2015) Study on the construction mechanism of stability lobes in milling process with multiple modes. Int J Adv Manuf Technol 79(1-4):589-603

24. Lin JS, Weng CI (1991) Nonlinear dynamics of cutting process. Int J Mech Sci 33(8):645

25. Zemzemi F, Rech J, Ben Salem W, Dogui A, Kapsa P (2009) Identification of a friction model at tool/chip/workpiece interfaces in dry machining of aISI4142 treated steels. J Mater Process Technol 209(8):3978-3990

26. Rech J, Claudin C, D'Eramo E (2009) Identification of a friction model-application to the context of dry cutting of an AISI 1045 annealed steel with a TiN-coated carbide tool. Tribol Int 42(5):738-744

27. Childs THC (2006) Friction modelling in metal cutting. Tribology in Manufacturing Processes 260(3):310-318

28. Stembalski M, Preś P, Skoczyński W (2013) Determination of the friction coefficient as a function of sliding speed and normal pressure for steel $\mathrm{c} 45$ and steel 40HM. Archives of Civil and Mechanical Engineering 13(4):444-448

29. San-Juan M, Martín Ó, Santos F (2010) Experimental study of friction from cutting forces in orthogonal milling. Int J Mach Tools Manuf 50(7):591-600

30. Nayfeh AH (1985) Problems in perturbation. John Wiley \& Sons, Inc

31. Rusinek R, Weremczuk A, Warminski J (2011) Regenerative model of cutting process with nonlinear Duffing oscillator. Mechanics and Mechanical Engineering 15(4):129-143 